\title{
Design and Development of a Real Time Virtualized Cloud Based Mobile Phones Data Storage for Ethio Telecom
}

\author{
Eshete Derb Emiru ${ }^{1}$, Walelign Tewabe Sewunetie ${ }^{2}$ \\ I'Department of Information Technology, College of Technology, Debre Markos University, Ethiopia, \\ ${ }_{2}^{2}$ (Department of Information Technology, College of Technology, Debre Markos University, Ethiopia,
}

\begin{abstract}
Mobile phone is one of the most widely and extensively used devices for communication across the world for both personal and business activities. The capabilities of mobile phones have been improving very quickly in terms of computing power, storage, feature support, and developed applications. Mobile Cloud Computing simply refers to an infrastructure where both the data storage and the data processing are made to happen outside of the mobile phones. With the advent of cloud services for mobile application, it has greatly enhanced the scalability and security. Hence, it stores contact numbers and messages sent from different mobile users in its memory and SIM card. However, if the mobile phone or the SIM card is lost or damaged due to different incidents, the information stored before can decidedly be lost. Such instant is very costly and risky for recovery of the lost information. Most cloud file storage providers offer limited free use and charge for additional storage once the free limit is exceeded. However, Ethio telecom customers don't have any means of using a mobile cloud computing services in order to share and access their files, documents, contacts, photos, videos. The reason behind the problem is lack of online payment system in Ethiopia. Even though various cloud computing model are existed, in this paper private cloud computing model is chosen which involves a distinct and secure cloud based environment in which only the specified customers can operate. In order to solve a problem a new mobile cloud computing architecture is designed through private cloud computing model. In effect, based on this architecture design, a new algorithm for Mobile users is developed so as to get unlimited, available and scalable data storage capacity.
\end{abstract}

Keywords: Cloud Computing, Ethio telecom, Mobile Cloud Computing (MCC), Mobile Phone, Mobile Cloud Storage, Private Cloud Computing Model.

\section{Introduction}

The Cloud Computing has emerged as a new computing paradigm which aims to provide reliable, customized, dynamic computing environments focused towards better quality of service and IT infrastructure availability without much financial burden. Mobile cloud computing is the form of cloud computing in combination with mobile Phones. Mobile Phones are increasingly becoming an essential part of human life as the most effective and convenient communication tools which is not restricted by time and place. The capabilities of mobile Phones have been improving very quickly in terms of computing power, storage, feature support, and developed applications. Mobile cloud applications move the computing power and data storage away from mobile phones and into the cloud, bringing applications and mobile computing to not just smartphone users but a much broader range of mobile subscribers [1]. Mobile cloud storage is a form of cloud storage that applies to storing an individual's mobile device data in the cloud and providing the individual with access to the data from anywhere. Mobile cloud storage additionally facilitates syncing and sharing data across multiple mobile phones such as tablets, iPhone, and Blackberry and Android smart phones. Ethio telecom, previously known as the Ethiopian Telecommunications Corporation (ETC), is an integrated telecommunications services provider in Ethiopia: providing internet, web service and telephone services using mobile Phones [1]. However, Ethio telecom is not giving mobile cloud storage application for every users due to absence of online payment service. This paper focused on design and development of real time private mobile cloud storage for Ethio telecom.

\subsection{Mobile Cloud Computing (MCC)}

\section{Related works}

Mobile cloud storage is a form of cloud storage that is accessible on mobile phones such as tablets, and smartphones. Mobile cloud storage providers offer services that allow the user to create and organize files, folders, music, and photos, similar to other cloud computing modals. Services are used by both individuals and companies. Most cloud file storage providers offer limited free use and charge for additional storage once the free limit is exceeded. These costs are usually charged as a monthly subscription rate and have different rates depending on the amount of storage desired. In 2009, cloud services revenue was about $\$ 58.6$ billion and in 2011 was about $\$ 2.6$ trillion. That revenue would top $\$ 152$ billion in 2014. Cloud computing revenue is 
expected to grow steadily around the world from 110.27 billion U.S. dollars in 2012 to more than 200 billion in 2016 [2]. The cloud services revenue increases due to the following advantages of mobile cloud computing.

A) Extending battery lifetime: Several solutions have been proposed to enhance the CPU performance [3], [4] and to manage the disk and screen in an intelligent manner [5], [6] to reduce power consumption. However, these solutions require changes in the structure of mobile Phones, or they require a new hardware that results in an increase of cost and may not be feasible for all mobile Phones. Computation offloading technique is proposed with the objective to migrate the large computations and complex processing from resource-limited Phones (i.e., mobile Phones) to resourceful machines (i.e., servers in clouds). This avoids taking a long application execution time on mobile Phones which results in large amount of power consumption.

B) Improving data storage capacity and processing power: Storage capacity is also a constraint for mobile Phones. MCC is developed to enable mobile users to store/access the large data on the cloud through wireless networks. First example is the Amazon Simple Storage Service (Amazon S3) [7] which supports file storage service. Another example is Image Exchange which utilizes the large storage space in clouds for mobile users [8]. This mobile photo sharing service enables mobile users to upload images to the clouds immediately after capturing. Users may access all images from any devices. With cloud, the users can save considerable amount of energy and storage space on their mobile Phones since all images are sent and processed on the clouds. Sites like Flickr [9] are also the successful mobile photo sharing applications based on MCC. Facebook is the most successful social network application today, and it is also a typical example of using cloud in sharing images. MCC also helps reducing the running cost for compute-intensive applications that take long time and large amount of energy when performed on the limited-resource devices. Cloud computing can efficiently support various tasks for data warehousing, managing and synchronizing multiple documents online. For example, clouds can be used for transcoding, playing chess [10], [11], or broadcasting multimedia services [12] to mobile Phones. In these cases, all the complex calculations for transcoding or offering an optimal chess move that take a long time when perform on mobile Phones will be processed quickly on the cloud. Mobile applications also are not constrained by storage capacity on the devices because their data now is stored on the cloud.

C) Improving reliability: Storing data or running applications on clouds is an effective way to improve the reliability since the data and application are stored and backed up on a number of computers. This reduces the chance of data and application lost on the mobile Phones. In addition, MCC can be designed as a comprehensive data security model for both service providers and users. For example, the cloud can be used to protect copyrighted digital contents (e.g., video, clip, and music) from being abused and unauthorized distribution [13] Also, the cloud can remotely provide to mobile users with security services such as virus scanning, malicious code detection, and authentication [14]. Also, such cloud-based security services can make efficient use of the collected record from different users to improve the effectiveness of the services.

\subsection{Secured Mobile Phones Data Storage}

The cloud is now much cheaper and mobile users prefer to store data in the cloud rather than storing in mobile phones. The huge amount of data can be stored on private clouds which are cheaply available which enables huge amount of data to be stored in cloud. Even the mobile is lost the data is safe in cloud and even the contacts. You have an ID to access the cloud you can access it from new mobile device too but the Authenticity, Authorization and Accountability is checked before accessing to the data. The data storage is made easier. There are more chances of mini phones in future, Mobile Cloud Computing allows to store data and retrieve the huge amount of data stored in cloud, the mobile device is merely an interface to access the data and making it a powerful device [15].

Backup software provided with most new mobile phones is secure enough, but if your PC is destroyed you can lose that information, so online backup services provide extra security for your contacts, emails, text messages, photos and documents, which can all be managed online to give you peace of mind [5]. Contacts, emails, documents and photos are all stored on a remote cloud server, meaning if your computer is lost, destroyed or stolen along with your mobile, your data is still available within minutes [16].

\section{Ethio Telecom}

Ethio telecom is owned by the Ethiopian government and maintains a monopoly over all telecommunication services in Ethiopia [1]. Ethio telecom was managed, on a management contract arrangement from 2010 to 2013 June, by France Télécom, and was required to comply with Ethiopian Government orders [17]. The government said it outsourced the management as ETC was not able to meet the demands of the fast-growing country. It also said that telecommunications services would not be privatized, at least not in the near future [18]. 
In 2015, there are over 7 billion mobile subscribers worldwide, up from 738 million in 2000 . There are 3.2 billion people using the internet, two billion of these live in developing countries [19]. However, those Ethio telecom customers don't have any means of using a mobile cloud computing services in order to share and access their files, documents, contacts, photos, videos. The reason behind the problem is lack of online payment system in Ethiopia.

\subsection{Private cloud service model}

\section{Proposed system}

A private cloud is a particular model of cloud computing that involves a distinct and secure cloud based environment in which only the specified client can operate. As with other cloud models, private clouds will provide computing power as a service within a virtualized environment using an underlying pool of physical computing resource. However, under the private cloud model, the cloud (the pool of resource) is only accessible by a single organization providing that organization with greater control and privacy [20]. A Private Cloud, or internal cloud, is used when the cloud infrastructure, proprietary network or data center, is operated solely for company or government departments who prefer to keep their data in a more controlled and secure environment, and serves customers within the business fire-wall [20]. The private cloud allows organizations to outsource the management of their IT infrastructure while retaining tighter control over the location and management of the resources. The price to pay for this is that the costs are likely to be higher because there is less potential for economy of scale, and resilience may be lower because of the limit on service resources available [20]. The technical mechanisms used to provide the different services which can be classed as being private cloud services can vary considerably and so it is hard to define what constitutes a private cloud from a technical aspect. Instead such services are usually categorized by the features that they offer to their client. Traits that characterise private clouds include the ring fencing of a cloud for the sole use of one organization and higher levels of network security. They can be defined in contrast to a public cloud which has multiple clients accessing virtualised services which all draw their resource from the same pool of servers across public networks. The features and benefits of private clouds therefore are [20]:

\section{A) Higher security and privacy:}

Public clouds services can implement a certain level of security but private clouds using techniques such as distinct pools of resources with access restricted to connections made from behind one organisation's firewall, dedicated leased lines and/or on-site internal hosting - can ensure that operations are kept out of the reach of prying eyes

\section{B) More control:}

As a private cloud is only accessible by a single organization, that organization will have the ability to configure and manage it inline with their needs to achieve a tailored network solution. However, this level of control removes somes the economies of scale generated in public clouds by having centralized management of the hardware

\section{C) Cost and energy efficiency:}

Implementing a private cloud model can improve the allocation of resources within an organization by ensuring that the availability of resources to individual departments/business functions can directly and flexibly respond to their demand. Therefore, although they are not as cost effective as a public cloud services due to smaller economies of scale and increased management costs, they do make more efficient use of the computing resource than traditional LANs as they minimize the investment into unused capacity. Not only does this provide a cost saving but it can reduce an organization's carbon footprint too

\section{D) Improved reliability:}

Even where resources (servers, networks etc.) are hosted internally, the creation of virtualized operating environments means that the network is more resilient to individual failures across the physical infrastructure. Virtual partitions can, for example, pull their resource from the remaining unaffected servers. In addition, where the cloud is hosted with a third party, the organization can still benefit from the physical security afforded to infrastructure hosted within data centers.

\section{E) Cloud bursting:}

Some providers may offer the opportunity to employ cloud bursting, within a private cloud offering, in the event of spikes in demand. This service allows the provider to switch certain non-sensitive functions to a public cloud to free up more space in the private cloud for the sensitive functions that require it. Private clouds 
can even be integrated with public cloud services to form hybrid clouds where non-sensitive functions are always allocated to the public cloud to maximize the efficiencies on offer.

\subsection{Ethio-Telecom Mobile Cloud Computing Data Storage}

Ethio telecom is the only ISP in Ethiopia and there is no way to accomplish any online payments like visa, PayPal, and others. So Ethio telecom customers are incapable to pay online and use MCC due to foreign currency exchange rate in addition to future challenges of security. Even though limited free storage given in different cloud services such as Apple's iCloud, Google Drive and Dropbox which are the most recognizable examples of mobile cloud storage in the globe, Ethio telecom customers problem solved by the newly designed architecture shown fig 1.

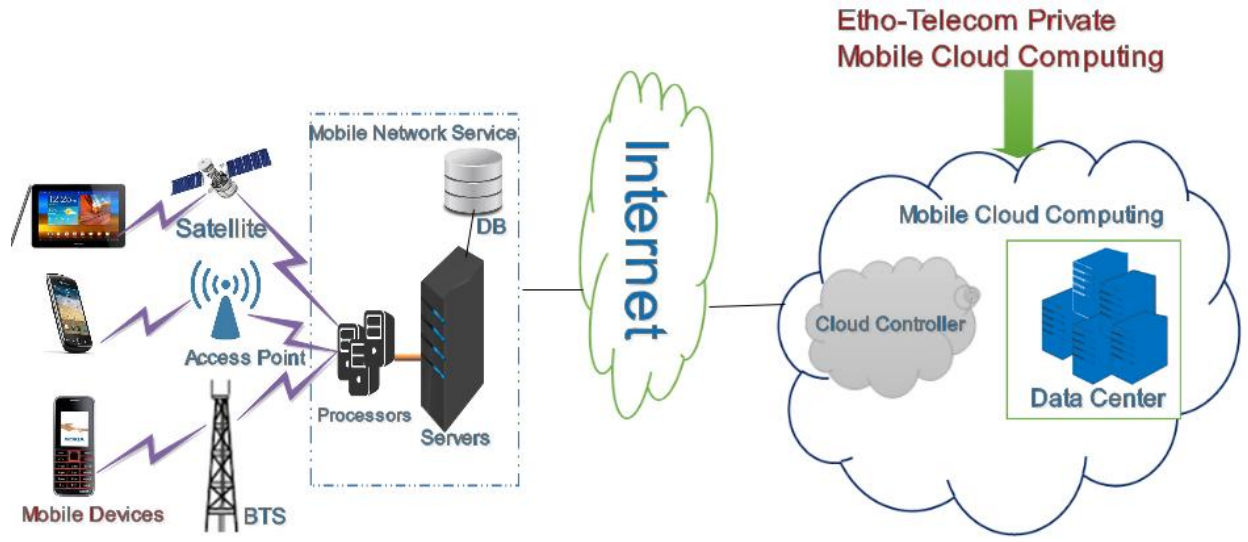

Figure 1.Ethio-Telecom Private Mobile Cloud Computing Architecture

To store and access data virtually in a real time manner the researchers create an algorithm which can be developed using android application and can be installed in android platform phones. The algorithm developed for the above Ethio telecom MCC architecture is indicated below.

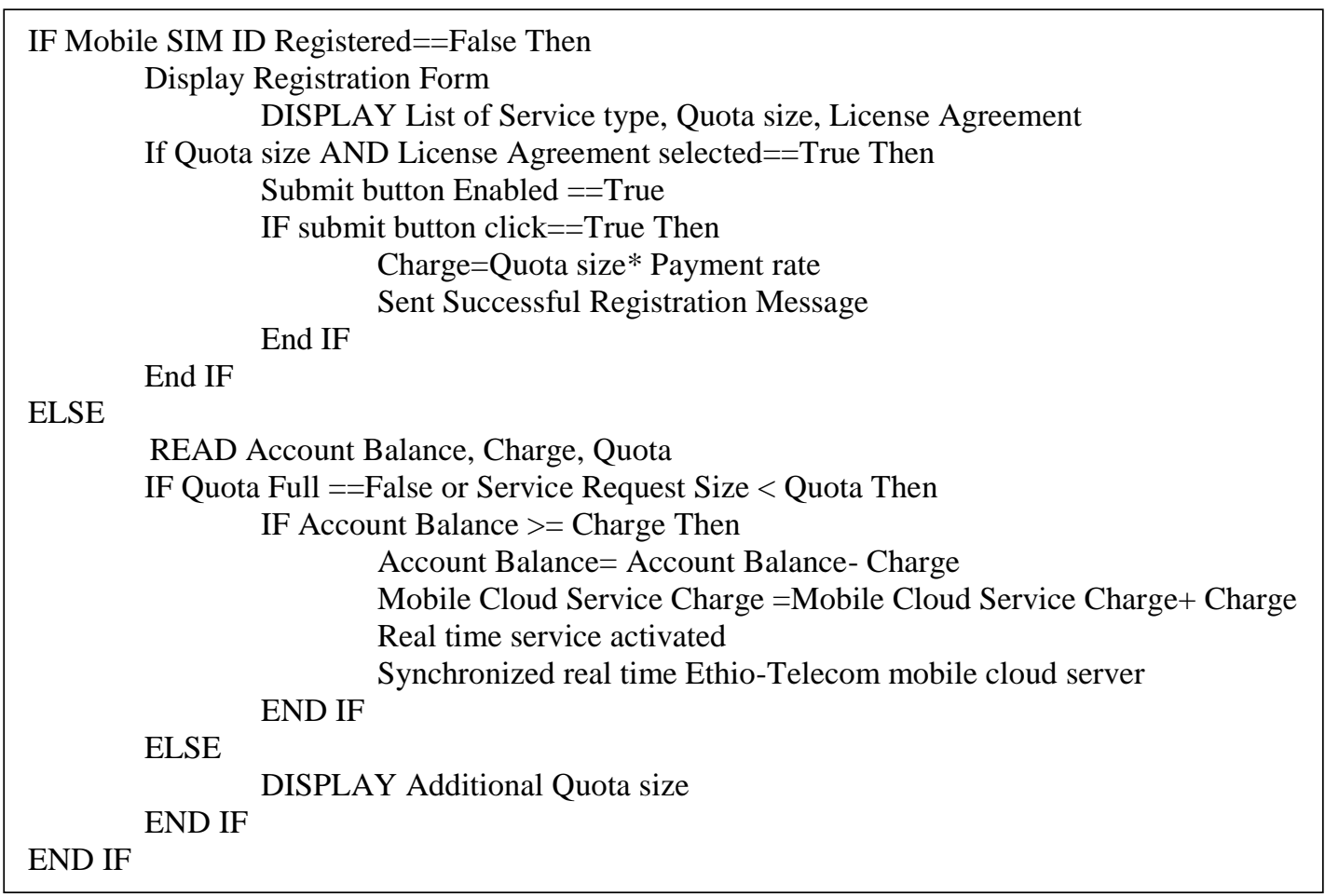

Algorithm 1: Ethio-Telecom Private Mobile Cloud Computing Algorithm

\section{Conclusion}

In this paper various literatures on mobile cloud computing were reviewed and the gaps to get real time cloud computing service in the context of Ethio telecom were identified. In order to solve a problem a new mobile cloud computing architecture was designed through private cloud computing model. Based on this 
architecture a new algorithm for Mobile users was developed so as to get unlimited, available and scalable data storage capacity.

\section{References}

[1]. S. Publishing, "The CIA World Factbook 2014," 2014. [Online]. Available: https://www.cia.gov/library/publications/the-worldfactbook/geos/et.html.

[2]. Statista, "global-revenue-generated-with-cloud-computing-since-2009," 2016. [Online]. Available: http://www.statista.com. [Accessed 15 July 2016].

[3]. K. R, "Low power design methodologies for mobile communication," in In Proceedings of IEEE International Conference on Computer Design: VLSI in Computers and Processors, 2003.

[4]. P. LD, "Low-power chips for high-powered handhelds," in IEEE Computer Society Magazine, 2003.

[5]. D. JW, "Power benchmark strategy for systems employing power management," in In Proceedings of the IEEE International Symposium on Electronics and the Environment, 2002.

[6]. Mayo RN, Ranganathan P, "Energy consumption in mobile devices: why future systems need requirements aware energy scaledown," in In Proceedings of the Workshop on Power-Aware Computing Systems, 2003.

[7]. "Amazon S3," [Online]. Available: https://aws.amazon.com/s3/. [Accessed 2016 July 15].

[8]. Oberheide J, Veeraraghavan K, Cooke E, Flinn J, Jahanian F, "Virtualized in-cloud security services for mobile devices," in In Proceedings of the 1st Workshop on Virtualization in Mobile Computing, 2008.

[9]. M. K.-V. Vartiainen E, "User experience of mobile photo sharing in the cloud," in In Proceedings of the 9th International Conference on Mobile and Ubiquitous Multimedia (MUM), 2010.

[10]. B. A. D.-k. C. e. a. Cuervo E, "MAUI: making smartphones last longer with code offload," in In Proceedings of the 8th International Conference on Mobile systems, applications, and services, 2010.

[11]. K. H. Garcia A, "Cloud transcoding for mobile video content delivery," in In Proceedings of the IEEE International Conference on Consumer Electronics (ICCE), 2011.

[12]. L. Y. Kumar K, "Cloud computing for mobile users," can offloading computation save energy.IEEE Computer Society, 2010.

[13]. Li L, Li X, Youxia S, Wen L, "Research on mobile multimedia broadcasting service integration based on cloud computing," in In Proceedings of the IEEE International Conference on Multimedia Technology (ICMT), 2010.

[14]. Zou P, Wang C, Liu Z, Bao D, "A cloud based drm scheme with SIM card," in In Proceedings of the 12th International AsiaPacific on Web Conference (APWEB), 2010.

[15]. M. Rani, "Mobile Cloud Computing and Its Challenges," International Advanced Research Journal in Science, Engineering and Technology, vol. 2, no. 2, pp. 68-73, December 2015.

[16]. A. K. A. C. W. Itani, "Energy-efficient incremental integrity for securing storage in mobile cloud computing," in Conference on Energy Aware Computing, ICEAC, Cairo, Egypt, Dec. 2010.

[17]. N. W. Hoboken, Attacks on the press journalism on the world's front lines, Committee to Protect Journalists (2013).

[18]. "Out of reach:Telecoms in Ethiopia," 24 August 2013. [Online]. Available: http://www.economist.com/news/middle-east-andafrica/. [Accessed 20 July 2016].

[19]. "ethiopia-aspires-to-raise-mobile-subscribers-in-five-years," [Online]. Available: http://www.2merkato.com. [Accessed 15 july 2016].

[20]. "What is a Private Cloud?," [Online]. Available: http://www.interoute.com/cloud-article. [Accessed 16 July 2016].

[21]. N. W. Hoboken, Attacks on the press journalism on the world's front lines, Committee to Protect Journalists (2013). , August 2014. 